\title{
Excreción Urinaria de Calicreína en Recién Nacidos
}

\author{
Dr. Fernando Olavarría U. ${ }^{1}$; Dr. Carlos Vío L. ${ }^{2}$; Dr. Sergio Krause H. ${ }^{1}$; \\ Dr. Fernando Hermann H. 1; E.U. Gema Santander M. ${ }^{3}$
}

\section{Kallikrein Excretion in Newborn Infants}

Kallikrein excretion and some parameters of renal function were studied on 20 , one day old, newborn infants (12 preterm and 8 term infants). Preterm infants excreted less kallikrein $(p<0.05$ ), had lower creatinine clearance ( $<<0.02$ ) and urinary osmolality $(\mathrm{p}<0.03$ ) and they showed also higher values of urinary volume $(\mathrm{p}<0.01$ ), $\mathrm{Fe}_{\mathrm{Na}}+(\mathrm{p}<0.01)$, Fe K $\mathrm{K}^{+}(\mathrm{p}<0.05)$, and free water clearace $(\mathrm{p}<0.02)$ than infants born at term. Kallikrem excretion related directly with gestational age $(p<0.01)$ and body weight $(p<0.03)$ and inversely with FeNa + $(p<0.04)$ and urinary volume $(p<0.01)$. When urinary kallikrein excretion was corrected to body surface these relations lost statistical significance, except with gestational age ( $p<0.04$ ). The values of kallikrein excretion in newborns were very low as compared to those of a young adult population. As kallikrein is synthesized in the distal nephron, we have advanced as an hypothesis that this could be a reflection of inmature distal tutbular mass and/or be caused by relative unresponsiveness of the distal nephron of newborn infants to hormones known to stimulate kallikrein in adults, such as aldosterone and antidiuretic hormone.

(Key words: Kallikrein excretion. Newborn infants. Term and preterm).

En los mecanismos de regulación de la función rena! se reconoce la participación de al menos tres sistemas hormonales vasoactivos, tales como el sistema renina-angiotensina-aldosterona, las prostaglandinas renales y el sistema calicreína-

1. Instituto de Pediatría, Facultad de Medicina, Universidad Austral de Chile.

2. Instituto de Fisiología, Facultad de Medicina, Universid ad Austral de Chile.

3. Servicio de Pediatría, Unidad de Neonatología, Hospital Regional de Valdivia.

Trabajo realizado con apoyo de la Dirección de Investigación y Desarrollo, Univ. Austral de Chile, Proyectos S-83-11 y 5-85-38 y Proyectos 1050/85 de FONDECYT. $\operatorname{cinina}^{1,2}$.

El estudio de estos sistemas en el riñón en vías de desarrollo ha despertado gran interés recientemente ${ }^{3}$, así, se han establecido altos niveles de aldosterona circulante y excreción urinaria aumentada de prostaglandinas en el periodo neonata $1^{4,5}$. No obstante, existe escasa información respecto al sistema calicreína-cinina en recién nacidos de diferente edad gestacional ${ }^{6,7}, 8$.

El presente estudio fue diseñado para evaluar la excreción renal de calicreina en recién nacidos de término y pretérmino durante las primeras horas de vida, y a través de sus relaciones con otros parámetros de la función renal, lograr un mejor conocimiento de la ontogenia del sistema calicreína y su participación en la fisiologia del riñón en desarrollo. 


\section{MATERIAL y METODO}

El estudio se efectuó en recién nacidos de diferente edad gestacional $y$ de sexo masculino por la mayor facilidad para recolección de orina. La edad gestacional fue asignada de acuerdo a historia menstrual y evalua. cjón pediátrica según pauta de Dubowitz y Dubowitz 9 .

Las deterninaciones se realizaron entre las 24 y 48 horas de vida. Durante ese período se aportó un volumen mínimo de $80 \mathrm{ml} \times \mathrm{kg} \times$ día como leche maternizada (NANß, Nestlé) vía oral yo solución glucosalina 1/4 normal por vía endovenosa. Para la evaluación de la función renal, se utilizó el método descrito previamente 10 .

Una alicuota del volumen urinario de $\mathbf{2} 4$ horas de cada recién nacido fue dializada durante la noche contra agua corriente y guardada en frío a $-20^{\circ} \mathrm{C}$ hasta su posterior análisis.

La actividad de calicreína fue medida por hidrólisís del sustrato sintético D-val-Leu-Arg-p- nitroanjlida (S-2266, Kabi, Sweden) de acuerdo a la técrica descrita por Amundsen y col.11. La actividad enzimática se expresó como unidades de calicreína amidasa (KUA) siendo una KUA la actividad enzimática capaz de hidrolizar 1 umol de sustrato por $\mathrm{ml}$, por minuto, a $37^{\circ} \mathrm{C}, \mathrm{pH} 8,2$ Cada muestra ind iv idual fue confrontada contra su propio blanco urinario conteniendo aprotinin (trasylol, Bayer) $1000 \mathrm{KIU} / \mathrm{ml}$.

Los resultados fueron expresados como mKUA $x$ día y también estand arizados a $1,73 \mathrm{~m}^{2}$ (mKUA $\mathrm{x}$ día $x$ $1,73 \mathrm{~m}^{2}$ ). La calicreírna urinaria fue además individualizada por su comportamiento en columna de cromatografía DEAE-Sephadex y su perfil de inhibición con inhibidores de tr ipsina ${ }^{12}$.

El análisis estadístico fue practicado en un computador Digital 2020, usando el programa S.P.S.S. Se utilizó la prueba $T$ de Student para muestras indepen. dientes y el análisis de correlación de Parson, fijandose la probabilidad de error en menos de un $5 \%$.
Se volicitó la autorización materna para la incorporación de cada recién nacido a este estudio.

\section{RESULTADOS}

Los 20 recién nacidos que comprenden este estudio, tenían una edad gestacional ( $x \pm$ D.E.) de $35,7 \pm 4,0$ semanas (rango 26 a 41 semanas) todos adecuados a edad gestacionat: con un peso de $2.364 .5 \pm 1.031,1 \mathrm{~g}$ (rango 840 a $4.080 \mathrm{~g}$ ), 8 eran recién nacidos de ténnino (RNT) y 12 eran de pretérmino (RNPT). Para este último grupo, la edad gestacional fue de 33.3 \pm 3.1 semanas.

El volumen aportado durante el periodo de estudio fue de $95,1 \pm 23,0 \mathrm{ml} \mathrm{x} \mathrm{kg} \mathrm{x} \mathrm{día} \mathrm{para} \mathrm{los}$ RNPT y de 95,6 $\pm 13,3 \mathrm{ml} \mathrm{x} \mathrm{kg} \times$ día para los RNT, sin existir diferencias signifjcativas entre ambos grupos.

En el grupo de los RNPT, 5 desarrollaron sindrome de dificultad respiratoria de moderada intensidad durante el periodo de estudio. No hubo diferencias significativas en los parámetros funcionales estudiados entre éstos y los que no evidenciaron trasiorno respiratorio, y por ende fueron considerados como un sólo grupo.

Con excepción de la ser̃alada, no hubo otras enfermedades o malformaciones evidentes que pudieran interferir los resultados de este estudio.

En la Tabla 1 se resumen los resultados del estudio de función renal y excreción urinaria de calicreina para los grupos RNT y RNPT.

Tabla 1

Función Renal y Excreción Urinaria de Calicreina en Recién Nacidas de Pretérmino y Término

\begin{tabular}{|c|c|c|c|c|c|c|c|}
\hline & \multicolumn{3}{|c|}{ Pretérmino } & \multicolumn{3}{|c|}{ Término } & \multirow[t]{2}{*}{$\mathrm{p}$} \\
\hline n & & 12 & & & 8 & & \\
\hline Peso (g) & 1.650 & \pm & $415 *$ & 3.443 & \pm & 788 & $<0,001$ \\
\hline $\begin{array}{l}\text { Volumen urinario } \\
(\mathrm{m} 1 \times \mathrm{kg} \times \mathbf{h})\end{array}$ & 3,34 & \pm & 1,0 & 1,13 & \pm & 0,4 & $<0,01$ \\
\hline $\begin{array}{l}\text { Depuración creatinina } \\
(\mathrm{ml} \times \text { min) }\end{array}$ & 1,68 & \pm & 0,63 & 3,55 & \pm & 1,60 & $<0,02$ \\
\hline $\mathrm{FeNa}^{+}(\%)$ & 0.96 & \pm & 0,82 & 0,13 & \pm & 0,10 & $<0,01$ \\
\hline $\mathrm{Fe} \mathbf{K}^{+}(\%)$ & 15,2 & \pm & 7,3 & 9,1 & \pm & 4,2 & $<0,05$ \\
\hline $\begin{array}{l}\text { Osmolarid ad urinaria } \\
\operatorname{mOSN} \times \mathrm{kg}-\mathrm{H}_{2} \mathrm{O}\end{array}$ & 117,1 & \pm & 31.7 & 194,8 & \pm & 78,8 & $<0,03$ \\
\hline Calicreíne urinaria & 2,88 & \pm & 1,07 & 9,49 & \pm & 5,73 & $<0,02$ \\
\hline $\operatorname{mKAU} x \operatorname{dia} \times 1,73 \mathrm{~m}^{2}$ & 37,35 & \pm & 13,01 & 82,72 & \pm & 52,78 & $<0,05$ \\
\hline $\begin{array}{l}\text { Depuración agua libre } \\
\text { ml x min. }\end{array}$ & 0,048 & \pm & 0,02 & 0,022 & \pm & 0,02 & $<0,02$ \\
\hline
\end{tabular}


Tabla 2

Correlación entre Excreción Urinaria de Calicreina y Edad Gestacional, Peso Corporal y algunos Parámetros de Función Renal.

Excreción urinaria

de calicreína M x KAU x día

\begin{tabular}{|c|c|c|}
\hline Versus & I & $\mathbf{p}$ \\
\hline Edad gestacional & 0,565 & $<0,01$ \\
\hline Peso & 0,433 & $<0,03$ \\
\hline $\begin{array}{l}\text { Depuración creatinina } \\
(\mathrm{m} / \mathrm{mj})\end{array}$ & 0,382 & 0,05 \\
\hline $\begin{array}{l}\text { Volumen urinario } \\
(\mathrm{ml} \times \mathrm{kg} x \mathrm{~h})\end{array}$ & $-0,509$ & $<0,01$ \\
\hline $\mathrm{FeNa}^{+}$ & $-0,420$ & $<0,04$ \\
\hline $\mathrm{Fe}_{\mathrm{K}}^{+}$ & $-0,376$ & n.s. \\
\hline $\begin{array}{l}\text { Depuración agua libre } \\
\text { (ml } x \text { min) }\end{array}$ & $-0,016$ & n. 8 . \\
\hline
\end{tabular}

n.8. = no significativo.

En comparación con el grupo de término, los RNPT tuvieron una depuración de creatinina significativamente menor y valores más bajos de osmolaridad urinaria. Referente a volumen urinario, excreción fraccional de sodio y potasio, y depuración de agua libre, los RNPT presentaron valores significativamente más altos que los RNT.

En cuanto a excreción urinaria de calicreína, los valores fueron significativamente más bajos en el grupo de RNPT, aún corregido por superficie corporal.

La excreción urinaria de calicreína se correlacionó directa y significativamente con edad gestacional, peso corporal y en forma negativa con volumen urinario y excreción fraccional de sodio $\left(\mathrm{Fe} \mathrm{Na}{ }^{+}\right.$). No se demostró correlación con excreción fraccional de potasio $\left(\mathrm{FeK}^{+}\right)$ni con depuración de agua libre; observándose una correlación directa, pero en el límite de lo significativo, con depuración de creatinina (Tabla 2).
Al corregir la excreción urinaria por superficie corporal (mKAU x día $\times 1,73 \mathrm{~m}^{2}$ ) estas correlaciones pierden significación estadística, a excepción de aquella establecida con edad gestacional (r 0,$421 ; \mathrm{p}<0,04$ ).

\section{DISCUSION}

La inmadurez del riñón al nacimiento se expresa fundamentalmente a través de una menor velocidad de filtración glomerular $10,13,14$, una limitada capacidad para reabsorber el sodio ultrafiltrado ${ }^{3-17}$ y una reducida habilidad para concentrar la orina ${ }^{18}$

El proceso de maduración renal es dependiente de la edad gestacional, como lo demuestra el hecho de una capacidad funcional renal más limitada en el RNPT en comparación con el RNT.

Nuestros resultados, con respecto a función renal, son concordantes con investigaciones previas en RNT y RNPT en periodo similar de la vida extrauterina $3,10,18,19$.

Poco se conoce sobre la excreción urinaria de calicreína en el recién nacido y de su participación en el proceso de maduración funcional renal. Recientemente, en este país, Saieh y col.20 estudiaron la excreción urinaria de calicreína en niños de diferentes edades, demostrando un ascenso progresivo de ella a partir de los seis meses da edad, para estabilizarse alrededor de los 2 años, lo que estaría señalando que la edad es un determinante del proceso madurativo de este sistema hormonal. En el mismo sentido apuntan nuestros resultados al demostrar una menor excreción urinaria en el grupo de RNPT en comparación con los RNP, lo que asociado a la buena correlación observada entre excreción urinaria de calicreína estandarizada a superficie corporal vs edad gestacional, estaria indicando que dicha excreción es un reflejo del proceso de crecimiento y maduración del riñón.

Los valores de excreción urinaria de caliçreina

Tabla 3

Comparación de Excreción Urinaria de Calicreína entre Recién Nacido de Pretérmino, Término y Adulto Joven.

\begin{tabular}{cccc}
\hline & R.N.P.T. & R.N.T. & Adulto Joven* \\
\hline$n$ & 12 & 8 & 14 \\
$\begin{array}{l}\text { Calicreina urinatia } \\
\text { m x KAU x dia } x 1,73 \mathrm{~m}^{2}\end{array}$ & $37,35 \pm 13,01^{* *}$ & $82,72 \pm 52,78$ & $1,680 \pm 140$
\end{tabular}

* Valores obtenidos en adultos jóvenes voluntarios participando en un estudio en el Instituto de Fisiología de la Universidad Austral de Chile.

$* * \vec{x} \pm$ D.E. 
en población adulta son veinte a cuarenta veces superiores a los encontrados en recién nacidos, aún corregidos por superficie corporal (Tabla 3), contrastando con los altos niveles de otras hor motras vasoactivas, tales como el sistema reninaangiotensina-aldosterona y la excreción aumentada de prostaglandinas renales en el periodo neonatal $3,4,5,7,21$.

Por lo general estos sistemas vasoactivos renales se modifican en un mismo sentido en yariadas condiciones clínicas y experimentales, entre ellas, el sindrome de Bartter ${ }^{2}$, el embarazo normal ${ }^{23}$, dieta baja en sodio ${ }^{24}$, hipoaldosteronismo hiporreninémico ${ }^{25}$ etc. Sin embargo, en el período neonatal esta tendencia estaría ausente, no quedando claro su significado según nuestros resulta. dos. Sólo se podría aventurar la hipótesis de que tal vez, en el momento de nacer, estos tres sistemas pudieran encontrarse en diferente estado de maduración.

Recientemente Vio y col., han postulado que la síntesis de calicreína renal ocurre en el del túbulo de conección del nefrón distal ${ }^{26}$ y de acuerdo a lo señalado por Sprago ${ }^{27}$, su excreción es un reflejo de masa tubular funcional del túbulo distal. Se sabe además que en su control participa un sistema multihormonal ${ }^{2 a}$. Entre las hommonas conocidas que estimulan la excreción de calicreína urinaria y que en parte podrían ser responsables de los bajos niveles obseryados en el recién nacido, deberían mencionarse a la aldosterona y la hormona antidiurética, que han demostrado tener un efecto estimulante de dicha excreción en ratas adultas 29,30 .

Numerosos estudios han establecido la discreta respuesta del nefrón distal a aldosterona en el rifón en desarrollo $16,17,21,31$, lo que de acuerdo a los trabajos de Robillard ${ }^{6}$ también se manifestaría en el estímulo de excreción de calicreina. Este autor, basado en estudios hechos en fetos de ovejas y ovejas recién nacidas, demostró que la excreción urinaria aumenta en el último período de gestación y más aún durante la vida extrauterina, indicando que el rango de excreción de calicreína es dependiente de los niveles de aldosterona y que la edad concepcional podria modular la sensibilidad renal a aldosterona.

Referente a hormona antidiuretica, se ha demostrado que las ratas recién nacidas tienen menor concentración de receptores para dicha hormona en el nefrón distal en comparación con ratas adultas ${ }^{32}$, y recientemente Aperia y col. ${ }^{33}$ han demostrado convincentemente que los RNT pueden incrementar su excreción acuosa frente a una ingesta aumentada de agua, a través de un mecanismo independiente de hormona antidiurética.
Entonces, es muy probable que siendo el nefrón distal el sitio de sintesis de calicreína, como to han demostrado los estudios ultraestructurales $^{26}$, los bajos niveles de excreción observados. pudieran ser un efecto de pobre respuesta del nefrón a aldosterona y hormona antidiurética $y$ un reflejo de masa tubular funcional del túbulo conector del nefrón distal.

\section{RESUMEN}

Se estudió la excreción urinaria de calicreina y algunos parámetros de función renal en 20 recièn nacidos (12 RNPT y 8 RNT) de sexo masculino. durante las 24 y 48 horas de vida. Con respecto a los RNT, los RNPT tuvieron excreción urinaria de calicreina más baja $(\mathrm{p}<0,05)$, menor depuración de creatinina $(p<0,02)$ y más baja osmo. laridad urinaria $(p<0,03$ ). Los RNPT tuvieton un volumen urinario más alto $(p<0.01)$. mayor excreción fraccional de sodio $(p<0,01)$. menor excrecion fraccional de potasio $(p<0,05)$ y mayor depuración de agua libre $(p<0,02)$. La excreción urinaria de calicreína se correlacionó directamente con edad gestacional $(p<0,01) y$ peso corporal $(p<0,03)$, e inversamente con excreción fraccional de sodjo $(p<0,04)$ y volumen urinario $(\mathrm{p}<0,01)$. Al corregir la excreción de calicreina a superficie corporal, las relaciones pierden significación estadística, excepto con edad gestacional $(p<0,04)$.

Los valores de calicreína urinaria en recién nacido fueron bajos comparados con los de población adulta. Dado que la sintesis de calicreína ocurre en el tuibulo distal, se plantea la hipótesis que esta menor excreción en la edad neonatal está relacionada con inmadurez funcional del túbulo, o menor respuesta del nefrón distal a homonas que se sabe estimulan la excreción de calicreian en el adulto, como aldosterona y hormona antidiurética, 0 ambas.

\section{REFERENCIAS}

1. Moyfield, R.K.: Margolitus H.S.: Renal kallikreinkinin system. Relation renal function and blood pressure. Am. J. J. Nephrol, 3: 145, 1983.

2. MCGiff, J.C. Vio, $C P$. Vasoactive peptides, prostaglandins and the kidney. NZJ. Med. 96: 883, 1983.

3. Spitzer, A., editor: The Kidney during Development. Morphology and Function. Proceedings of the First International Workshop on Deyelopmental Renal Physiology. October 1980. Masson Publishing USA, New York 1982.

4. Dilton, M.J.; Gillon, M.E.A.; Kyness, J.M.; de Swiet. $M$ : Plasma renin activity and aldosterane concentration in the human newborn. Arch. Dis. Child. 51 : $537,1976$. 
5. Scherer, B.: Joppich, R.: Krauss, H., Wever, P.C.: Postnatal Development on the renal prostaglandins system as followed by urinary excretion in human neonates, En: Spitzer, A. (editor): The Kidney during Development, New Yotk 1982; Masson Publishing USA:pg. 151-157.

6. Robillard, J.E.; Lawton, W.J.; Weisman, A., Sessions, $C$.: Developmental aspect of the renal kallikrein-like activity in fetal and newborn lambs. Kidney Int. 22: 594, 1982.

7. Godard, C.: Valloton, M.B.; Favre, L.: Urinary prostaglandins, vasopressin and kallikrein excretion in healthy children from birth to adolescence. J. Pedjatr, 100: 898, 1982.

8. Betkerur, M.V.; Yeh, T.F.; Miller, K., Glosser, R.J.: Pildes, R.S.: Indomethacin and its effect on renal function and urinary kallikrein excretion in premature infants with patent ductus arterious. Pediatrics 68: 99,1981 .

9. Dubowitz, L.M.; Dubowitz, V,; Goldberg, C.: Clinical assessment of gestational age in the newborn infant. J. Pediatr. 77: 1, 1970 .

10. Olavarria, F.; Krause, S.; Barranco, $L$. : Harding, $C$.; López, M.I.: Función renal en recién nacidos de término y preténnino durante el segundo día de vida. Rev. Chil. Pediatr. 57: 39, 1986.

11. Amuhdsen, E.: Putter, J.; Friberger, P.: Know, M.; Larsbraten, $M_{+}$Cleason, $G_{+}$: Methods for the determination of glandular kallikrein by means of a chromogenic tripeptide substrate. Adv. Exp. Med. Biol. 120: 83, 1979.

12. Vio, C.P.: Churchill, L.: Rabito, S.F.: Terragno, A.: Carretero, O.A.: Terragno, N.A.: Renal kallikrein in venous effluent of filteting and non-filtering isolated kidneys. Adv. Exp. Med. Biol. 156: 897, 1983.

13. Guignard, J.P.; Torrado, A ; Da Cunha, O.; Gautier, $E$.: Glomerular fultration rate in the first three weeks of like. J. Pediatr. 87: 268, 1975.

14. Sertel, $H_{\text {.; }}$ Scopes, $J_{\text {.: }}$ Rates of creatinine clearance in babie less than one week of age. Arch. Dis. Child. 48: $717,1973$.

15. Aperia, A.: Broberger, O.; Thadenius, K.: Zetterström, $R$.: Development of tenal control of salt and fluid homeostasis during the first year of life. Acta Paediatr. Scand. 64: 393, 1975.

16. Sulyok, E., Varga, F.: Jobst, K., Csaba, J.F.: On the mechanism of renal sodium handling in newborn infants. Biol. Neonate $37: 75,1980$.

17. Spitzer, A.: The role of the kidney in sodium homeostasis during maturation. Kidney Int. 21: $539,1982$.

18. Guignard, JP.: Renal function in the newborn infant. Pediatr. Clin. North Am. 29: 777, 1982.

19. Aperia, A.; Broberger, O.: Elinder, G.; Thodenius, K.: Zetterström, R.: Postnatal changes in glomerular filtration rate in preterm and fullterm infants. En: Spitzer, A. (editor): The Kidney during Develop- ment, New York, 1982; Masson Publishing USA: pag. 133-137.

20. Sajeh, $C$.: Valdes, $G$,; Tobar, $M$; Chacón, $C_{*}$ Croxatto, $\boldsymbol{H}$ : Excreción de calicreína urinaria en niños sanos a distintas edades. Rev. Chil. Pediatr. $56: 345,1985$.

21. Sulyok, E.: Nemeth, M.: Teny, I. Csaba, IF., Györy, E.: Ertl, T.: Varga, F.: Postnatal development of Renin-Angiotensin Aldosterone System (RAAS), in relation to electrolyte bajance in premature infants. Pediatr, Res. 13: 817, 1979.

22. Hallushka, P.V.; Wohltmann, M.; Privitera, P.J. Hurwitz, G.: Margoluis, H.S.: Barter's syndrome; Urinary prostaglandin E-like material and kallikrein: Indomethacin effects. Ann. Intern. Med. 87: 281, 1977.

23. Valdés, G., Espinoza, P.; Moore, R.: Croxatto, H.R.: Urinary kallikrem and plasma tenin activity in normal human pregnancy. Hypertension 3 (Suppl I1): 55,1981 .

24. Margolius, H.S.; Honwitz, D.; Geller, R.G.; Alexander, R.W.; Gill, J.R.: Pisano, J.J.: Keiser, H.R. Lrinary kalliksein excretion in normal man: Relationships to sodium intake retining steroids. Circulation Res. 35: 812, 1974.

25. Mezzono, S.; Gonzólez, J.; Hott, H.; Vio, C.P.; Urinary kallikrein activity in the hiporeninemic hypoaldosteronism syndrome. Rev. Med. Chil. 112: $364,1984$.

26. Vio, C.P.; Figueroa, C.D.: Subcellular localization of renal kallikrein by ultrastructural immunocytochemistry. Kidney Int. 28: 36, 1985

27. Spragg, $J_{. ;}$Denmey, D.L.: Tilney, N.L.; Austen, K.F.: Kallikrein excretion in renal trasplant recipients and in uninephrectomized donors. Kidney Int. 28: 75, 1985 ,

2B. Marin-Grez, M.: Multihormonal regulation of renal kallikrein. Biochem. Pharmacol. 31: 3941, 1982.

29. Margolius, H.S., Chao, $J$,; Kaizu, T.: The effects of aldosterone and spironolactone on tenal kallikrein in the rat. Clin. Sci, 51:279, 1976.

30. Feies-Toth, G.: Zohajszky, $T_{\text {.i }}$ Filep, $J .:$ Effect of vasopressin on renal kallikrein excretion. Am. J. Physiol. 239: F 388, 1980.

31. Rodriguez-Soriano, J.; Vallo, A.; Oliveros, $R$. ef al: Renal handling of sodium in premature and fullterm neonates: a study using slearance methods during water diuresis. Pedia1r. Res. 17: 1013, 1983.

32. Rajerison, R.B.; Butlen, $D$, Jand, $S$.: Ontogenic development of antidiuretic hormone receptors in Iat kidney. comparison of homonal binding and adenylate cyclase activation. Mol. Cell. Endocrinol. 4: $271,1976$.

33. Aperio, A.: Herin, P.; Lundin, S.: Melin, $P$. Zetterström. $R .:$ Regulation of renal water excretion in newborn fullterm infants. Acta Pediatr. Scand, 73: 717, 1984. 